\title{
REVIEW
}

\section{Electrical Stimulation as a Means for Improving Vision}

\author{
Amer Sehic, ${ }^{*}$ Shuai Guo, ${ }^{\dagger}$ Kin-Sang Cho, ${ }^{\ddagger}$ Rima M. Corraya, ${ }^{\dagger}$ Dong F. Chen, ${ }^{\ddagger}$ and Tor P. Utheim ${ }^{\star \dagger} \S \Phi$
}

From the Department of Oral Biology, * Faculty of Dentistry, University of Oslo, Oslo, Norway; the Department of Medical Biochemistry, ${ }^{\dagger}$ Oslo University Hospital, Oslo, Norway; the Department of Ophthalmology, ${ }^{\ddagger}$ Schepens Eye Research Institute, Massachusetts Eye and Ear, Harvard Medical School, Boston, Massachusetts; the Department of Ophthalmology, ${ }^{\complement}$ Drammen Hospital, Vestre Viken Hospital Trust, Drammen, Norway; and the Faculty of Health Sciences, University College of South East Norway, Notodden, Norway

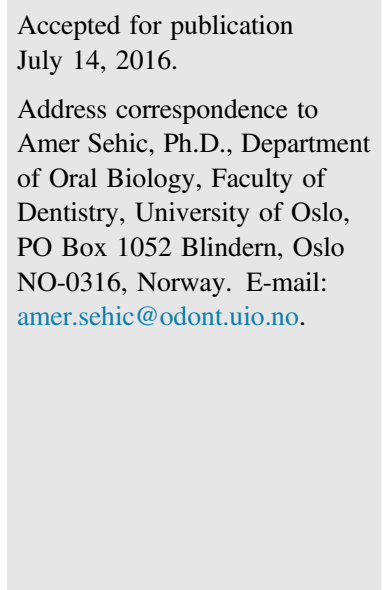

Accepted for publication

July 14, 2016

Address correspondence to Amer Sehic, Ph.D., Department of Oral Biology, Faculty of Dentistry, University of Oslo, PO Box 1052 Blindern, Oslo NO-0316, Norway. E-mail: amer.sehic@odont.uio.no.

\begin{abstract}
Evolving research has provided evidence that noninvasive electrical stimulation (ES) of the eye may be a promising therapy for either preserving or restoring vision in several retinal and optic nerve diseases. In this review, we focus on minimally invasive strategies for the delivery of ES and accordingly summarize the current literature on transcorneal, transorbital, and transpalpebral ES in both animal experiments and clinical studies. Various mechanisms are believed to underlie the effects of ES, including increased production of neurotrophic agents, improved chorioretinal blood circulation, and inhibition of proinflammatory cytokines. Different animal models have demonstrated favorable effects of ES on both the retina and the optic nerve. Promising effects of ES have also been demonstrated in clinical studies; however, all current studies have a lack of randomization and/or a control group (sham). There is thus a pressing need for a deeper understanding of the underlying mechanisms that govern clinical success and optimization of stimulation parameters in animal studies. In addition, such research should be followed by large, prospective, clinical studies to explore the full potential of ES. Through this review, we aim to provide insight to guide future research on ES as a potential therapy for improving vision. (Am J Pathol 2016, 186: 2783-2797; http://dx.doi.org/10.1016/j.ajpath.2016.07.017)
\end{abstract}

\section{Eye Diseases and Treatment Options}

Blindness affects 39 million people worldwide, and its prevalence continuously increases with an aging population (http://www.who.int/mediacentrelfactsheets/fs282/en). The leading causes of irreversible blindness in the developed world include glaucoma, age-related macular degeneration (AMD), diabetic retinopathy, and retinitis pigmentosa (RP). Glaucoma refers to a group of ocular diseases that result in damage to the optic nerve, leading to progressive, irreversible loss of retinal ganglion cells, ${ }^{1}$ and it is the second leading cause of blindness worldwide. ${ }^{2}$ It is estimated that by 2020 , approximately 11 million individuals will be blind from glaucoma. ${ }^{2}$ Current glaucoma therapies, including medication, laser trabeculoplasty, surgery, or a combination of any of these, are directed solely at lowering intraocular pressure, which slows vision loss for some patients but cannot restore vision loss due to disease. AMD is another progressive disease leading to blindness as a result of photoreceptor cell loss in the macula (ie, the central part of the retina). It primarily affects central vision, which is critical for daily activities, such as reading and driving. AMD is the third leading cause of blindness in the world and the primary cause of visual deficiency in industrialized countries. $^{3}$ Clinically, AMD is divided into two forms: wet and dry. There are currently several treatment options for wet AMD. However, no established therapy is available for dry

\footnotetext{
Supported by the Department of Oral Biology (University of Oslo), the Department of Medical Biochemistry (Oslo University Hospital), and NIH/ National Eye Institute grants R41 EY025913, R01 EY025259, and P30 EY03790-33.

D.F.C. and T.P.U. contributed equally as senior authors.

Disclosures: None declared.
} 
AMD, which constitutes $85 \%$ to $90 \%$ of known cases. Certain treatments, including intravitreal injection of antivascular endothelial growth factor, have shown considerable success in slowing disease progression and reducing the number of blind people worldwide by preventing further neovascularization from wet AMD. However, no welldocumented cures exist that can reverse damage that has already been inflicted to the photoreceptors. RP encompasses several genetic defects that result in photoreceptor loss and blindness. The prevalence of RP is reported to be $1: 3000$ to $1: 5000 .{ }^{4}$ In contrast to AMD, the retina is first affected peripherally, with gradual progression to the central retina. RP targets rod photoreceptors, thus initially causing poor peripheral and night vision.

Although there are no currently available cures for these potentially blinding conditions (eg, glaucoma, RP, and AMD), there are many fields of research that give hope of visual restoration to patients with end-stage eye disease. Visual cycle modulators that mediate the intracellular processing of vitamin A are currently being applied in clinical trials for RP, and initial evidence has shown that they slow progression of disease and may provide better vision in patients with mild-to-moderate disease. ${ }^{5}$ Gene therapy aims at treating, curing, or preventing disease by delivering genes into the eyes; however, current therapies are primarily experimental, with most human clinical trials still in the research phase. ${ }^{6}$ For patients only retaining light perception, optogenetic (ie, the use of genetic methods combined with optical technology to achieve retinal neuron function) and stem cell therapies are promising strategies for improving or restoring vision.

\section{ES in 0phthalmology}

The first-reported electrical stimulation (ES) of the eye to generate visual sensations to light was performed in 1755 by Charles LeRoy, who applied an electrical discharge on the eye surface of a patient blind from cataracts. The patient reported that the ES provoked strong flashes of light or phosphene. In 1873, Henri Dor reported the beneficial effects of ES on the eye via treatment of amblyopia, amauroses, glaucoma, retinochoroiditis, and white optic atrophy. ${ }^{8}$ In 1929 , Otfrid Foerster, a German neurosurgeon, reported a neural response to ES in a patient's visual cortex. ${ }^{9}$ In 1974 , the ES of the visual system was shown to elicit visual perceptions using suprathreshold stimulation. ${ }^{10}$ Thirty years later, the therapeutic potential of subthreshold ES of the retina was identified by Chow et al. ${ }^{11}$ The authors showed that patients having inactive subretinal chips generating only subthreshold currents exhibited visual improvement in areas far from the site of the electrical device, suggesting a generalized neuroprotective effect of ES. This finding was associated with the expression of neurotrophic factors and sparked a research interest in the effects of ES in animal experiments and thereafter in clinical studies for treating blindness.

\section{ES: Methods of Delivery}

During the past 10 years, there has been a significant increase in research on developing an ES treatment for various eye diseases. The basis of the therapeutic strategy is to stimulate neurons along the visual pathway with electrical current. Presently, there are eight routes by which ES has been delivered to patients with the purpose of improving vision: transpalpebral, transorbital, transcorneal, subretinal, epiretinal, and transchoroidal approaches, as well as the direct stimulation of the optic nerve or brain (Figure 1). Ocular stimulation targets the remaining retinal cells, whereas stimulation of the brain targets the higher visual structures. Although ocular stimulations are minimally invasive and simple to implement, stimulation of the brain is useful when connections between the eye and brain are affected, such as in advanced glaucoma.

\section{Retinal Prostheses}

Retinal prostheses (alias retinal implants or chips) use energy converters to generate electricity to mimic photoreceptor activities. Epiretinal implants electrically target the ganglion cell layer, subretinal implants primarily target the inner nuclear layer of the retina, and transchoroidal implants stimulate the retina from the outer region. ${ }^{12}$ Patients with RP usually exhibit defective pigment epithelium and photoreceptors, but the inner retina and ganglion cell layer are intact. To restore visual function, retinal prostheses must detect light, converting light energy into electricity and thereby activating downstream retinal neurons. The field of visual prosthetics has progressed significantly since the first implantation in the $1960 \mathrm{~s}^{13}$; however, many challenges must still be overcome before it can effectively restore vision in blind patients. ${ }^{12}$

\section{Minimally Invasive ES}

Transpalpebral, transorbital, and transcorneal ES (TES) have the advantage of being minimally invasive, only touching the skin and the cornea, with minor adverse effects reported. The use of these approaches reduces the risk of severe complications compared to retinal prostheses. It is of utmost importance that ES is minimally invasive; therefore, the choice of electrodes must be taken into consideration. In both animal experiments and clinical studies, TES has been widely adapted, and the contact lens electrodes are most commonly used. Until now, three types of electrodes have been used in TES: Dawson-Trick-Litzkow (DTL)-Plus electrodes, electroretinography (ERG)-Jet electrodes, and golden ring electrodes, the last lacking a contact lens. DTLPlus and ERG-Jet electrodes have been used in clinical studies and only negligibly stimulated different areas of the retina. In animal experiments, the ERG-Jet electrode is mostly used because of the simplicity of the procedure for fastening the electrodes onto animals. Transpalpebral 


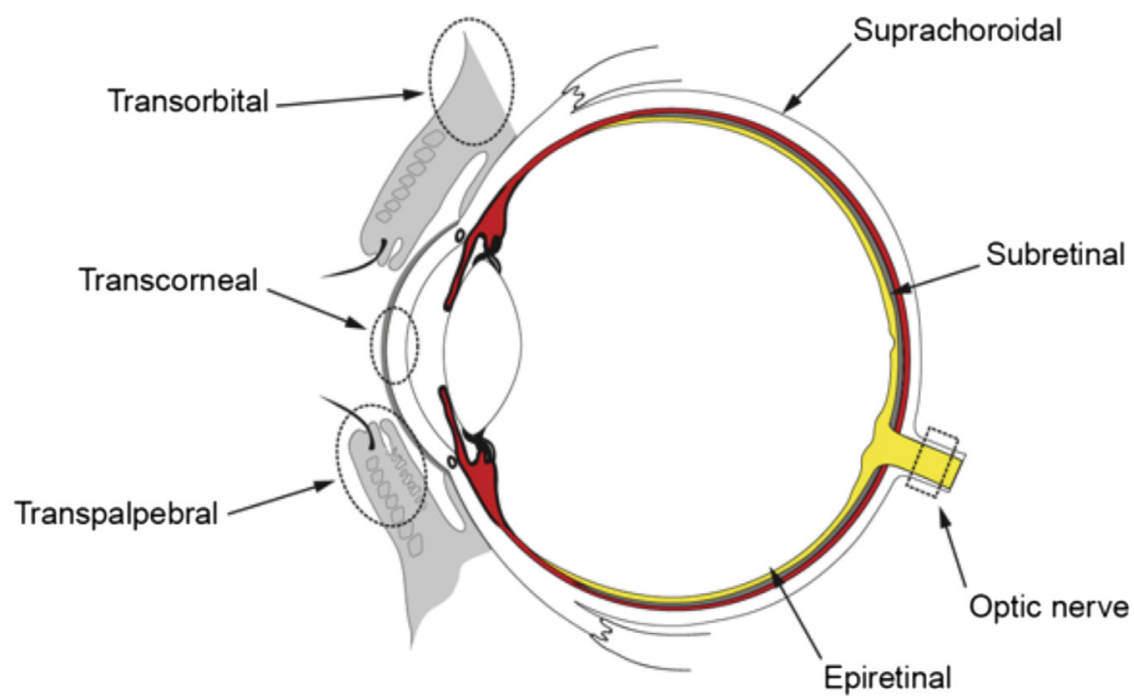

Figure 1 Different routes of electrical stimulation of the eye. The red circle represents choroidea, except for the anterior part of the eye. The gray semicircle represents the retinal pigment epithelial cells, and the yellow semicircle illustrates the retina. Image is courtesy of Håkon Raanes (Norway). (electrodes placed on the eyelids) and transorbital (electrodes placed at or near the eyeball) ES are other minimally invasive procedures. In the present review, we will systematically discuss the experimental populations and parameters, evaluation methods, major findings, advances, and challenges of the various ES procedures.

\section{Animal Experiments}

Many research groups have shown favorable effects of ES on the retina and optic nerve using rats, cats, and rabbits as experimental animals. So far, there are no animal studies on transpalpebral ES. However, beneficial effects of ES have been shown in one study using transorbital $\mathrm{ES}^{14}$ and in several animal experiments using TES (Table 1). ${ }^{15-35}$ These studies can be categorized as follows: i) healthy animals ${ }^{17-19,23,26,31,34}$; ii) transgenic animals ${ }^{22,28}$; iii) animals as a disease model for $\mathrm{RP}^{21}$; iv) animals with induced ischemic insult, ${ }^{27,29,33}$ optic nerve crush, ${ }^{15,16,20,32}$ and transected optic nerve ${ }^{24,25}$; and v) animal cells isolated from the retina. ${ }^{35}$

\section{TES in Animal Models}

In 2005, Morimoto et $\mathrm{al}^{24}$ observed that the survival of retinal ganglion cells (RGCs) was preserved from degeneration after transection of the optic nerve in rats, and that the degree of the electric charge was important for the rescue of cells. It was also demonstrated that insulin-like growth factor 1 (IGF-1) in the retina was up-regulated after stimulation. IGF-1 was initially strongly expressed in Müller cells and then also in the inner retina. In another study, the current intensity, frequency, pulse duration, waveform, and number of TES sessions were changed systematically. ${ }^{25}$ This vigorous approach demonstrated repeated stimulation after optic nerve transection to be the most advantageous. Furthermore, the highest neuroprotective effect on RGCs was achieved using the following stimulation parameters: 1 and 2 milliseconds per phase, 100 and $200 \mu \mathrm{A}$, frequency of 1.5 and $20 \mathrm{~Hz}$, and at least a 30-minute duration.

Enhanced survival of RGCs has also been observed after optic nerve crush, ${ }^{15,16,20,32}$ after light-induced retinal damage, ${ }^{26}$ and in ischemic rat retinas. ${ }^{33}$ Tagami et al ${ }^{32}$ revealed that the increased survival of RGCs was in accordance with the number of TES applications and that the daily application of TES exhibited the most effect. Furthermore, daily TES administration resulted in the up-regulated expression of IGF-1 to a greater extent than a single application of TES. Finally, enhanced regeneration of RGC axons by daily application of TES was completely blocked by a specific antagonist to the IGF-1 receptor, whereas the promotion of surviving RGCs was not. ${ }^{32}$

In a study involving Royal College of Surgeons rats, a generally used model for inherited retinal degeneration such as RP, the researchers demonstrated that retinal degenerative processes are markedly slowed by TES. TES increased survival of photoreceptors, as suggested by a significantly greater thickness of the outer nuclear layer, preserved ERG b-wave, and impaired retinal function in Royal College of Surgeons rats. ${ }^{21}$ In a study by Schatz et al, ${ }^{36}$ TES before mild light exposure temporarily preserved b-wave amplitudes and outer segment length, and reduced photoreceptor cell death after 2 weeks. The effects observed in this study were minor compared to the effects reported by Ni et al, ${ }^{26}$ where TES sessions were repeated and different electrode configurations were used. In a rabbit model of rhodopsin mutations that mimic RP, TES resulted in both increased photoreceptor survival and improved retinal function. ${ }^{22}$ In $\mathrm{P} 23 \mathrm{H}$ transgenic rats, delivery of TES twice per week for 12 weeks resulted in the significant preservation of photoreceptor sensitivity, whereas no histological changes accompanied the stimulation. ${ }^{28}$ A possible explanation for the unchanged morphology may be the low electrical intensity applied in this study. 


\section{TES and Changes in Brain Activity}

The beneficial effects of TES are not limited to rescuing neurons in the retina. Recently, Sergeeva et $\mathrm{al}^{31}$ successfully generated a new method for TES and flicker light stimulation in freely moving rats as well as a setup to record field potentials from the visual cortex and subcortical structures. A considerable advantage of this novel animal model, compared to what was described in previous studies, ${ }^{16,21,24,30}$ was the possibility to stimulate nonanesthetized rats. Another major advantage of this new animal model was that the rats were freely moving, permitting behavioral testing while simultaneously investigating the electrophysiological effects of stimulation. It was shown that the narcosis strongly influenced brain oscillations and thus may have interfered with TES or its effects on the brain. This was in accordance with a recent study in which TES-induced electroencephalogram aftereffects were only achieved while stimulating shallow (late) narcosis but not a deep (early) slow-wave stage. ${ }^{30}$ The visual responses in the cortex caused by TES were primarily detected in the areas representing the peripheral visual field. ${ }^{18}$ In this study, the authors showed that the responses were followed by intensive evoked field potentials. When increasing the pulse width or intensity of TES current, the gradual extension of the responsive regions from the cortical areas to areas representing the central visual field was observed. ${ }^{18}$

\section{Transorbital ES in Animal Experiments}

Compared to TES, the therapeutic effects and physiological mechanisms of transorbital alternating current stimulation are less well studied and are poorly understood. To our knowledge, there is only one published animal experiment that studied the effect of transorbital alternating current stimulation and characterized the responses in several compartments of the visual system. ${ }^{14}$ The findings from this study suggested that early stages of visual processing in generating electrically evoked potentials after transorbital alternating current stimulation were important, further proposing that the coordination of the input from the retina to the tectum and thalamus was an essential, functional mechanism in transorbital alternating current stimulation. ${ }^{14}$ These suggestions are in accordance with the results presented by Sergeeva et $\mathrm{al}^{30}$ demonstrating that the structural integrity at early stages of visual processing was essential for the after-effects achieved by TES.

\section{Clinical Studies}

\section{The Effects of TES in Clinical Studies}

There are 12 published clinical studies on the use of TES (Table 2). ${ }^{36-47} \mathrm{~A}$ controlled, randomized, and partially masked short-term study included 24 patients with RP and demonstrated significant improvements in visual field area and $b$-waves of the scotopic ERG. ${ }^{36}$ The study divided patients into three groups, depending on the strength of stimulation in relation to the individual phosphene threshold $(0 \%, 66 \%$, or $150 \%)$. The results showed that all functional parameters were slightly improved or remained constant in the $150 \%$ group, whereas there was no marked tendency in the $66 \%$ group. Compared to normal subjects, Morimoto et $\mathrm{al}^{41}$ showed that patients with RP and cone-rod dystrophy demonstrated significantly higher electrical thresholds (ie, T1, threshold current for initial phosphene; T2, threshold for eliciting a phosphene extending into the central field; and $\mathrm{P}$, threshold for a relative pupillary constriction $\geq 3 \%$ ). The authors concluded that TES could be used as one of the most essential tests to select candidates for retinal prostheses. In a study by Inomata et al, ${ }^{39}$ where three patients with long-standing retinal artery occlusion were treated, TES was found to improve both visual acuity and visual field. Moreover, multifocal ERGs demonstrated that the implicit time and amplitudes of all component waves significantly reformed after the TES treatment, suggesting that TES exhibited favorable effects on both the inner and outer retinal neurons in patients with retinal artery occlusion.

In another study of eight patients with long-standing or fresh branch retinal artery occlusion, improvements of both multifocal ERG and visual field parameters were reported after TES in long-standing cases, although improvements in the visual field were not observed in patients with fresh occlusion. ${ }^{44}$ Naycheva et $\mathrm{al}^{43}$ demonstrated that the slopes of the scotopic a-waves significantly increased in patients with central or branch retinal artery occlusion. TES treatment performed $>4$ months after the onset of nonarteritic ischemic optic neuropathy exhibited improved visual acuity in two of three eyes examined. ${ }^{38}$ Although visual acuity became impaired some months after the patients stopped receiving ES, after a second TES treatment, the vision improved again. This finding suggests a causal relationship between the treatment and visual improvement. In a recent study using TES, electrical phosphene threshold varied between disease groups. ${ }^{42}$ Across all tested frequencies (3, 6, 9, 20, 40, and $80 \mathrm{~Hz}$ ), electrical phosphene thresholds were significantly higher when compared with healthy subjects, except in Stargardt disease patients at $20 \mathrm{~Hz}$. Improvements in visual acuity in a recent case report with best vitelliform macular dystrophy suggest that TES should also be investigated in other cases of retinal dystrophies. ${ }^{45}$ Furthermore, after TES, the phosphene threshold current was markedly higher in patients with retinal degeneration compared with control subjects. ${ }^{47}$

It has been shown that TES (up to $150 \mu \mathrm{A}, 20 \mathrm{~Hz}, 30$ minutes, one session) increases choroidal blood flow in normally sighted patients, with minimal effects on the general blood circulation and intraocular pressure. ${ }^{40}$ The authors suggested that this is probably an important mechanism that underlies the beneficial effects of TES in 
Table 1 TES: Animal Experiments

\begin{tabular}{|c|c|c|c|c|c|c|}
\hline Reference & $\begin{array}{l}\text { Species; disease } \\
\text { model }\end{array}$ & $\begin{array}{l}\text { Experimental } \\
\text { population; } \\
\text { stimulation } \\
\text { location }\end{array}$ & Control group & ES parameters & Evaluation method & Results \\
\hline $\begin{array}{l}\text { Henrich-Noack } \\
\text { et al, } 2013^{15}\end{array}$ & $\begin{array}{l}\text { Male hooded rats; } \\
\text { induced ONC }\end{array}$ & $\begin{array}{l}16 \text { rats; cornea; } \\
\text { one eye }\end{array}$ & Other eye & $\begin{array}{l}100 \mu \mathrm{A}, 1 \text { millisecond/phase, } \\
\text { directly after } 0 \mathrm{NC} \text { and on } \\
\text { days } 3,7,11,15,19, \text { and } \\
23 \text { after } 0 \mathrm{NC}, 30 \text {-second } \\
\text { duration; delivered at 10, } \\
12,9,11,8,10,9 \text {, and } 12 \\
\mathrm{~Hz} \text { with } 5 \text {-second break } \\
\text { between each }\end{array}$ & ICON; ERG recordings & $\begin{array}{l}\text { TES inhibits post-traumatic } \\
\text { cell death considerably; } \\
\text { TES decreases ONC- } \\
\text { associated neuronal } \\
\text { shrinkage and swelling, } \\
\text { exceptionally in long-term } \\
\text { surviving RGCs }\end{array}$ \\
\hline $\begin{array}{l}\text { Henrich-Noack } \\
\text { et al, } 2013^{16}\end{array}$ & $\begin{array}{l}\text { Male hooded rats; } \\
\text { induced ONC }\end{array}$ & $\begin{array}{l}\text { Eight rats; } \\
\text { cornea; } \\
\text { both eyes }\end{array}$ & $\begin{array}{l}\text { Eight rats; } \\
\text { sham }\end{array}$ & $\begin{array}{l}100 \mu \mathrm{A}, 20-\mathrm{Hz} \text { biphasic } \\
\text { square-wave pulses of } 1 \\
\text { millisecond/phase, } \\
60 \text {-minute duration, } \\
\text { stimulated immediately } \\
\text { after } 0 \mathrm{NC} \text { and on day } 11 \\
\text { after induction of } 0 \mathrm{NC}\end{array}$ & $\begin{array}{l}\text { ICON before } O N C \text { and on } \\
\text { postlesion days } 3,7 \text {, and } \\
\text { 15; ERG recordings }\end{array}$ & $\begin{array}{l}\text { TES resulted in increased } \\
\text { surviving of RGCs; the rate } \\
\text { of } \mathrm{RGC} \text { death was more } \\
\text { variable in TES group; } \\
\text { changes in morphology of } \\
\text { survived neurons were } \\
\text { milder in TES group }\end{array}$ \\
\hline $\begin{array}{l}\text { Kanamoto et al, } \\
2015^{17}\end{array}$ & Wistar rats & $\begin{array}{l}18 \text { tats; cornea; } \\
\text { left eye }\end{array}$ & Right eye & $\begin{array}{l}50 / 100 / 200 \mu \mathrm{A}, 20 \mathrm{~Hz} \text {, } \\
\text { 1-millisecond pulses, } \\
\text { 30-minute duration, one } \\
\text { session }\end{array}$ & $\begin{array}{l}\text { Enucleated eyes } 30 \text { minutes } \\
\text { or } 24 \text { hours after TES; } \\
\text { proteomic analysis of } \\
\text { retina }\end{array}$ & $\begin{array}{l}25 \text { Functional proteins } \\
\text { exhibited higher levels of } \\
\text { expression in TES group } \\
\text { compared with control } \\
\text { group }\end{array}$ \\
\hline Ma et al, $2014^{18}$ & Healthy adult cats & $\begin{array}{l}13 \text { cats; cornea; } \\
\text { right eye }\end{array}$ & - & $\begin{array}{l}\text { In optical imaging } \\
\text { experiments: } 1.2 \mathrm{~mA} \text {, } \\
\text { 5-100 Hz, 2-40- } \\
\text { millisecond pulses/phase, } \\
\text { 2-second duration; } \\
\text { studying effects of } \\
\text { different parameters: } \\
0.24-2.64 \mathrm{~mA}, 20 \mathrm{~Hz} \text {, } \\
\text { 10-millisecond pulse width } \\
\text { per phase, } 1.2 \mathrm{~mA}, 20 \mathrm{~Hz} \text {, } \\
\text { 2-22 millisecond pulse } \\
\text { width per phase }\end{array}$ & $\begin{array}{l}\text { Multiwavelength optical } \\
\text { imaging of intrinsic } \\
\text { signals; } \\
\text { electrophysiological } \\
\text { recordings; hemoglobin } \\
\text { oxygenation; cerebral } \\
\text { blood volume }\end{array}$ & $\begin{array}{l}\text { OIS activation regions in the } \\
\text { visual cortex were } \\
\text { correlative with TES; } \\
\text { ascending current } \\
\text { intensities and longer } \\
\text { pulse width increased the } \\
\text { extent of activation; } \\
\text { largest response observed } \\
\text { at } 10-20 \mathrm{~Hz} \text {; TES led to } \\
\text { activation of peripheral } \\
\text { retina }\end{array}$ \\
\hline $\begin{array}{l}\text { Mihashi et al, } \\
2011^{19}\end{array}$ & Healthy adult cats & $\begin{array}{l}\text { Two cats; } \\
\text { cornea; } \\
\text { both eyes }\end{array}$ & - & $\begin{array}{c}\text { Maximum current up to } 1 \mathrm{~mA} \text {, } \\
20 \mathrm{~Hz}, 20 \text { paired biphasic } \\
\text { pulses of } 5 \text { milliseconds }\end{array}$ & $\begin{array}{l}\text { Electrophysiological } \\
\text { recordings; fundus images }\end{array}$ & $\begin{array}{l}\text { TES activates the RGCS; TES } \\
\text { changes the blood flow }\end{array}$ \\
\hline $\begin{array}{l}\text { Miyake et al, } \\
2007^{20}\end{array}$ & $\begin{array}{l}\text { Long-Evans rats; } \\
\text { induced ONC }\end{array}$ & $\begin{array}{r}\text { Five rats; } \\
\text { cornea }\end{array}$ & Five rats; sham & $\begin{array}{l}500 \mu \mathrm{A}, 20 \mathrm{~Hz} \text {, pulses, } \\
50 \text {-microsecond duration; } \\
\text { started immediately after } \\
\text { the } 0 \mathrm{NC} \text {, applied for } 6 \\
\text { hours }\end{array}$ & VEP; histology & $\begin{array}{l}\text { TES instantly increased VEP } \\
\text { amplitude, which was } \\
\text { weakened by the ONC; } \\
\text { increased number of axons } \\
\text { in retina observed } \\
\text { centrally beyond the } \\
\text { crushed region when } \\
\text { compared with the control } \\
\text { group }\end{array}$ \\
\hline $\begin{array}{l}\text { Morimoto et al, } \\
2007^{21}\end{array}$ & $\begin{array}{l}\text { RCS rats; model for } \\
\text { RP }\end{array}$ & $\begin{array}{l}18 \text { rats; cornea; } \\
\text { left eye }\end{array}$ & Right eye & $\begin{array}{c}50 \text { or } 100 \mu \mathrm{A}, 20 \mathrm{~Hz}, 1 \\
\text { millisecond, } 1 \text {-hour } \\
\text { duration, one session }\end{array}$ & ERG recordings; histology & $\begin{array}{l}\text { Compared to sham, } 100 \mu \mathrm{A} \\
\text { TES increased the } \\
\text { thickness of the ONL at } \\
\text { each time point; TES } \\
\text { resulted in increased } \\
\text { number of surviving } \\
\text { photoreceptors and } \\
\text { inhibited functional } \\
\text { reduction of retina }\end{array}$ \\
\hline $\begin{array}{l}\text { Morimoto et al, } \\
2012^{22}\end{array}$ & $\begin{array}{l}\text { Rhodopsin P347L } \\
\text { transgenic } \\
\text { rabbits }\end{array}$ & $\begin{array}{c}\text { Six rabbits; } \\
\text { cornea; } \\
\text { left eye }\end{array}$ & Right eye & $\begin{array}{l}700 \mu \mathrm{A}, 20 \mathrm{~Hz}, 10 \\
\text { milliseconds/phase, } \\
\text { 1-hour duration, once a } \\
\text { week for } 6 \text { weeks }\end{array}$ & $\begin{array}{l}\text { ERG recordings; histology; } \\
\text { immunohistochemistry }\end{array}$ & $\begin{array}{l}\text { TES led to increased survival } \\
\text { of photoreceptors and } \\
\text { preserved ERGs }\end{array}$ \\
\hline $\begin{array}{l}\text { Morimoto et al, } \\
2014^{23}\end{array}$ & Healthy adult cats & $\begin{array}{c}\text { Eight cats; } \\
\text { cornea; } \\
\text { left eye }\end{array}$ & - & $\begin{array}{l}\text { Biphasic pulses: } 0.1,0.5,1.0, \\
2.0 \mathrm{~mA}, 20 \mathrm{~Hz} \text { at } 20 \text { pulses; } \\
\text { pulse durations: } 0.5,1.0, \\
2.0,3.0,5.0,10.0 \\
\text { milliseconds/phase at } 20 \\
\mathrm{~Hz} \text { at } 20 \text { pulses; } \\
\text { frequencies of } 5,10,20, \\
30,50 \mathrm{~Hz} \text { at } 5\end{array}$ & $\begin{array}{l}\text { Optical imaging of reflectance } \\
\text { changes in retina; } \\
\text { electrophysiological } \\
\text { recordings from OC }\end{array}$ & $\begin{array}{l}\text { The intensity increased with } \\
\text { the frequency, pulse } \\
\text { duration, and current } \\
\text { intensity; TES resulted in } \\
\text { the reflective changes on } \\
\text { the optic disk and retinal } \\
\text { vessels, and in activation } \\
\text { of retinal neurons }\end{array}$ \\
\hline
\end{tabular}

(table continues) 
Table 1 (continued)

\begin{tabular}{|c|c|c|c|c|c|c|}
\hline Reference & $\begin{array}{l}\text { Species; disease } \\
\text { model }\end{array}$ & $\begin{array}{l}\text { Experimental } \\
\text { population; } \\
\text { stimulation } \\
\text { location }\end{array}$ & Control group & ES parameters & Evaluation method & Results \\
\hline & & & & $\begin{array}{l}\text { milliseconds/phase at } 20 \\
\text { pulses; duration of } 0.5,1 \text {, } \\
4 \text {, and } 5 \text { milliseconds/ } \\
\text { phase at } 50 \mathrm{~Hz}\end{array}$ & & \\
\hline $\begin{array}{l}\text { Morimoto et al, } \\
2005^{24}\end{array}$ & $\begin{array}{l}\text { Adult Wistar rats; } \\
\text { transected } 0 \mathrm{~N}\end{array}$ & $\begin{array}{l}26 \text { rats; cornea; } \\
\text { both eyes }\end{array}$ & 12 Rats; sham & $\begin{array}{l}100 \mu \mathrm{A}, 20 \mathrm{~Hz}, 3 \\
\text { milliseconds/phase, } \\
\text { 1-hour duration, one } \\
\text { session }\end{array}$ & $\begin{array}{l}\text { Quantification of RGC } \\
\text { density; RT-PCR; Northern } \\
\text { blotting; Western blotting; } \\
\text { immunohistochemistry }\end{array}$ & $\begin{array}{l}\text { TES increased the number of } \\
\text { surviving axotomized } \\
\text { RGCs; the electric charge } \\
\text { strength had important } \\
\text { role for the degree of } \\
\text { rescue; IGF-1 was up- } \\
\text { regulated in the retina } \\
\text { after TES; IGF-1 was first } \\
\text { expressed in the Müller } \\
\text { cells and then diffused } \\
\text { into the inner retinal layer }\end{array}$ \\
\hline $\begin{array}{l}\text { Morimoto et al, } \\
2010^{25}\end{array}$ & $\begin{array}{l}\text { Wistar rats; } \\
\quad \text { transected ON }\end{array}$ & $\begin{array}{l}36 \text { rats; cornea; } \\
\text { left eye }\end{array}$ & $\begin{array}{l}12 \text { Rats; sham; } \\
\text { right eye }\end{array}$ & $\begin{array}{l}0.5,1,2,3, \text { and } 5 \\
\text { milliseconds/phase, with } \\
100 \mu \mathrm{A}, 20 \mathrm{~Hz} \text { for } 60 \\
\text { minutes; } 50,100,200, \\
300, \text { and } 500 \mu \mathrm{A} \text { with } 1 \\
\text { millisecond/phase, } 20 \mathrm{~Hz} \text {, } \\
60 \text { minutes; } 0.5,1,5,20, \\
50 \text {, and } 100 \mathrm{~Hz} \text { at } 100 \mu \mathrm{A}, \\
1 \text { millisecond/phase for } 60 \\
\text { minutes; } 15,30, \text { and } 60 \\
\text { minutes at } 100 \mu \mathrm{A}, 1 \\
\text { millisecond/phase, and } 20 \\
\mathrm{~Hz} \text {; repeated stimulations }\end{array}$ & $\begin{array}{l}\text { Quantification of RGC density } \\
\text { with fluorescence } \\
\text { microscope }\end{array}$ & $\begin{array}{l}\text { The optimal neuroprotective } \\
\text { parameters were } 1 \text { and } 2 \\
\text { milliseconds/phase, } 100 \\
\text { and } 200 \mu \mathrm{A} \text {, frequency of } \\
1,5, \text { and } 20 \mathrm{~Hz} ;>30 \\
\text { minutes of TES was } \\
\text { required to achieve a } \\
\text { neuroprotective effect; } \\
\text { repeated stimulation was } \\
\text { more neuroprotective than } \\
\text { a single stimulation } 14 \\
\text { days after transection }\end{array}$ \\
\hline
\end{tabular}

Ni et al, $2009^{26}$ SD rats

Osako et al, $2013^{27}$

SD rats; induced NAION

$\begin{array}{cccc}\begin{array}{c}\text { Rahmani et al, } \\ 2013^{28}\end{array} & \begin{array}{c}\text { P23H transgenic } \\ \text { rats; mimic } \\ \text { autosomal } \\ \text { dominant RP }\end{array} & \begin{array}{c}\text { Six rats; } \\ \text { cornea, } \\ \text { left eye }\end{array} & \begin{array}{c}10 \text { Rats, sham, } \\ 16 \text { weeks of } \\ \text { age }\end{array} \\ \begin{array}{c}\text { Schatz et al, } \\ 2012^{29}\end{array} & \begin{array}{c}\text { SD rats; induced } \\ \text { NAION }\end{array} & \begin{array}{c}15 \text { rats; cornea; } \\ \text { right eye }\end{array} & \begin{array}{c}15 \text { rats; sham; } \\ \text { 3 rats for } \\ \text { histology }\end{array}\end{array}$

19 rats; cornea 14 Rats; sham

cornea;

both eyes
Light

stimulation

$\mathrm{Hz}$; repeated stimulations on days $0,4,7$, and 10

after the transection were compared with a single stimulation 14 days after transection

$100-500 \mu \mathrm{A}, 20-100 \mathrm{~Hz}, 3$ milliseconds, 1.5 hours, one session; 200 or 300 $\mu \mathrm{A}, 20 \mathrm{~Hz}, 3$ milliseconds, one session per 3 days for up to 14 days

$100 \mu \mathrm{A}, 20 \mathrm{~Hz}, 1$ millisecond/ OCT measurements; STR phase, 60-minute duration; TES applied 3 hours after the induction of NAION and was later performed on the 1st, 4th, 7th, 14th, and 28th day

ERG recordings; histology; immunohistochemistry; RT-PCR; Western blotting
Post-TES exhibited an enhanced and longer-term protective effect than preTES; after TES, expression of $B C l-2$ was up-regulated, $B a x$ was down-regulated, and levels of CNTF and BDNF were up-regulated The use of TES resulted in decreased STR amplitude and the number of RGCs in NAION rats
TES led to preservation of b-wave amplitudes and rod sensitivity; no histological changes were associated with TES treatment

TES resulted in lower b-wave; photoreceptor cell death was observed in sham group, primarily in the superior hemiretina; eyes treated with TES exhibited preservation of $\mathrm{ONL}$ thickness and resulted in reduced death of photoreceptor cells 


\begin{tabular}{|c|c|c|c|c|c|c|}
\hline Reference & $\begin{array}{l}\text { Species; disease } \\
\text { model }\end{array}$ & $\begin{array}{l}\text { Experimental } \\
\text { population; } \\
\text { stimulation } \\
\text { location }\end{array}$ & Control group & ES parameters & Evaluation method & Results \\
\hline $\begin{array}{l}\text { Sergeeva et al, } \\
2015^{31}\end{array}$ & Lister hooded rats & $\begin{array}{l}24 \text { rats; cornea; } \\
\text { both eyes }\end{array}$ & - & $\begin{array}{l}300 \mu \mathrm{A}, \text { biphasic } 2- \\
\quad \text { millisecond pulses, } 1 \text { per } \\
\text { second, 20-minute } \\
\text { duration }\end{array}$ & $\begin{array}{l}\text { Exposed to flicker light; VEP } \\
\text { and EER in visual cortex } \\
\text { and superior colliculus }\end{array}$ & $\begin{array}{l}\text { Physiological responses } \\
\text { recorded after TES }\end{array}$ \\
\hline $\begin{array}{l}\text { Tagami et al, } \\
2009^{32}\end{array}$ & $\begin{array}{l}\text { Wistar rats; induced } \\
\text { ONC }\end{array}$ & $\begin{array}{l}36 \text { rats; cornea; } \\
\text { both eyes }\end{array}$ & 12 Rats; sham & $\begin{array}{l}100 \mu \mathrm{A}, 20 \mathrm{~Hz}, 1 \text { millisecond/ } \\
\text { phase, } 1 \text {-hour duration; } \\
\text { four protocols: a single } \\
\text { application on day } 0 \text {, two } \\
\text { on day } 0 \text { and } 7 \text {; four on day } \\
0,4,7 \text {, and } 10 \text {; and daily } \\
\text { applications on days } 0-12\end{array}$ & $\begin{array}{l}\text { Quantification of axonal } \\
\text { growth and RGC density; } \\
\text { IGF-1 immunostaining of } \\
\text { retina }\end{array}$ & $\begin{array}{l}\text { Daily applied TES encourages } \\
\text { both survival of RGCS and } \\
\text { regeneration of axons after } \\
\text { the crush of ON; TES } \\
\text { resulted in increased IGF-1 } \\
\text { immunoreactivity }\end{array}$ \\
\hline $\begin{array}{l}\text { Wang et al, } \\
2011^{33}\end{array}$ & $\begin{array}{l}\text { SD rats; ischemic } \\
\text { insult }\end{array}$ & $\begin{array}{l}28 \text { rats; cornea; } \\
\text { both eyes }\end{array}$ & Sham & $\begin{array}{l}300 \mu \mathrm{A}, 20 \mathrm{~Hz}, 3 \\
\text { milliseconds/phase, } 1 \text { hour } \\
\text { every } 2 \text { days up to } 14 \text { days }\end{array}$ & $\begin{array}{l}\text { ERG recordings; histology; } \\
\text { confocal laser microscope; } \\
\text { Western blotting; } \\
\text { immunohistochemistry }\end{array}$ & $\begin{array}{l}\text { Higher density of RGCs in TES } \\
\text { treated retinas on day } 7 \\
\text { and } 14 \text { after ischemia; TES } \\
\text { greatly preserved the } \\
\text { thickness of separate } \\
\text { retinal layers }\end{array}$ \\
\hline $\begin{array}{l}\text { Willmann et al, } \\
2011^{34}\end{array}$ & $\begin{array}{l}\text { Wild-type brown } \\
\text { Norway rats }\end{array}$ & $\begin{array}{l}17 \text { rats; cornea; } \\
\text { right eye }\end{array}$ & 16 rats; sham & $\begin{array}{l}200 \mu \mathrm{A}, 20 \mathrm{~Hz}, 1 \text { millisecond/ } \\
\text { phase, } 1 \text {-hour duration, } \\
\text { one session }\end{array}$ & $\begin{array}{l}\text { Microarray analysis; RT-PCR; } \\
\text { histology; morphometric } \\
\text { analysis; ERG recordings }\end{array}$ & $\begin{array}{l}490 \text { Genes exhibited different } \\
\text { expression after TES; } \\
\text { neuroprotective genes } \\
\text { such as Bax or TNF family } \\
\text { members were up- } \\
\text { regulated; ERG recordings } \\
\text { demonstrated } \\
\text { physiological retinal } \\
\text { function after TES }\end{array}$ \\
\hline
\end{tabular}

Bcl-2, B-cell lymphoma-2; BDNF, brain-derived neurotrophic factor; CNTF, ciliary neurotrophic factor; EEG, electroencephalogram; EER, electrically evoked response; ERG, electroretinogram; ES, electrical stimulation; ICON, in vivo confocal neuroimaging; IGF-1, insulin growth factor-1; LDH, lactate dehydrogenase; NAION, nonarteritic ischemic optic neuropathy; OC, optic chiasma; OCT, optical coherence tomography; OIS, optical imaging of intrinsic signals; 0N, optic nerve; ONC, optic nerve crush; ONL, outer nuclear layer; RCS, Royal College of Surgeons; RGCs, retinal ganglion cells; RP, retinitis pigmentosa; SD, SpragueDawley; STR, scotopic threshold response; TES, transcorneal electrical stimulation; VEP, visual evoked potentials; -, not reported.

ischemic retinal diseases. Another study demonstrated that TES at a frequency of $20 \mathrm{~Hz}$ evoked pupillary reflexes in subjects with normal vision, whereas biphasic pulse trains at a duration of 0.5 to 1.0 milliseconds and $20-$ to $50-\mathrm{Hz}$ frequencies evoked phosphene. ${ }^{37}$ Accordingly, both DTL-Plus and ERG-Jet electrodes showed visual cortex activation in association with the reported phosphene. ${ }^{46}$

\section{Transorbital and Transpalpebral ES in Clinical Trials}

There are six published studies on the use of transorbital ES $(\text { Table } 3)^{48-53}$ and three on the use of transpalpebral ES
(Table 3). ${ }^{54-56}$ In seven patients with chronic prechiasmatic visual system damage, it was found that transorbital ES strengthened $\alpha$ band functional connectivity, which was associated with perceptual improvements. Therefore, it is speculated that vision loss in the blind is caused not only by primary tissue damage but also by breakdown of synchronization in brain networks. ${ }^{48}$ In addition, transorbital ES in patients with optic nerve damage showed significant increases in detection ability, visual field, and visual acuity. ${ }^{49-52}$ All three published studies on the use of transpalpebral ES were performed in patients with AMD (Table 3). ${ }^{54-56}$ Transpalpebral ES was found to be safe in 
Table 2 TES: Clinical Studies

\begin{tabular}{|c|c|c|c|c|}
\hline Reference & Disease & $\begin{array}{l}\text { Experimental population; } \\
\text { stimulation location }\end{array}$ & Control group & ES parameters \\
\hline $\begin{array}{l}\text { Schatz et al, } \\
2011^{36}\end{array}$ & RP & $\begin{array}{l}24 \text { patients; cornea; } \\
\text { both eyes }\end{array}$ & Sham & $\begin{array}{l}\text { Intensity } 66 \% \text { and } 150 \% \\
\text { of EPT, } 20 \mathrm{~Hz}, 5- \\
\text { millisecond biphasic } \\
\text { pulses, } 30 \text { minutes per } \\
\text { week, for } 6 \text { weeks }\end{array}$ \\
\hline $\begin{array}{l}\text { Fujikado et al, } \\
2007^{37}\end{array}$ & Normal-sighted & Eight patients & - & $\begin{array}{l}25-250 \mu \mathrm{A} \text {, biphasic } \\
\text { pulses at } 5-50 \mathrm{~Hz}, 20 \\
\text { pulses of } 10 \text {-millisecond } \\
\text { duration }\end{array}$ \\
\hline $\begin{array}{l}\text { Fujikado et al, } \\
2006^{38}\end{array}$ & NAION; TON & $\begin{array}{l}\text { Eight patients; NAION } \\
\qquad(n=3) ; \text { TON }(n=5) ; \\
\text { cornea; one eye }\end{array}$ & Fellow eyes & $\begin{array}{l}300-800 \mu \mathrm{A}, 20 \mathrm{~Hz}, 10 \\
\text { milliseconds, } 30 \\
\text { minutes, one session }\end{array}$ \\
\hline $\begin{array}{l}\text { Inomata et al, } \\
2007^{39}\end{array}$ & RAO & $\begin{array}{l}\text { Three patients, two with } \\
\text { central and one with } \\
\text { branch RA0, cornea; } \\
\text { one eye }\end{array}$ & Fellow eyes & $\begin{array}{l}\text { 0-1100 } \mu \mathrm{A} \text { until evoking } \\
\text { phosphene, } 20 \mathrm{~Hz} \text {, } \\
30 \text {-minute duration, } \\
\text { once per month for up } \\
\text { to } 3 \text { months }\end{array}$ \\
\hline $\begin{array}{l}\text { Kurimoto et al, } \\
2010^{40}\end{array}$ & Normal-sighted & $\begin{array}{l}10 \text { patients; cornea; } \\
\text { both eyes }\end{array}$ & $\begin{array}{l}\text { Fellow eyes; } \\
\text { sham }\end{array}$ & $\begin{array}{c}\text { Up to } 150 \mu \mathrm{A}, 20 \mathrm{~Hz}, 30 \\
\text { minutes, one session }\end{array}$ \\
\hline
\end{tabular}

Evaluation method

VA; VF; ERG recordings; color discrimination; EPTs; blood tests

Measurement of PR pulses at $5-50 \mathrm{~Hz}, 20$ pulses of 10-millisecond duration
EEPR; BCVA; VF

VA; VF; mfERGs

LSFG; SBR; IOP; measurement of blood pressure and pulse rate

$50 \mu \mathrm{A}-2 \mathrm{~mA}$, biphasic pulses at $20 \mathrm{~Hz}, 20$ pulses of 10-millisecond duration; three electrical current thresholds: T1, $\mathrm{T} 2$, and $\mathrm{P}$ thresholds

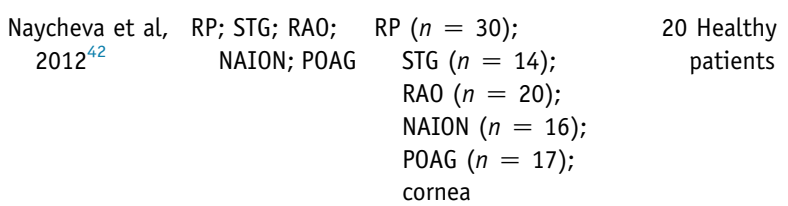
20 Healthy
patients

Naycheva et al, Central and $2013^{43}$ branch RAO
13 patients; 12 with central, 1 with branch sham RAO
$1 \mu \mathrm{A}-10 \mathrm{~mA}, 3,6,9,20$,

\begin{tabular}{|c|c|c|}
\hline $\begin{array}{c}\text { Oono et al, } \\
2011^{44}\end{array}$ & Branch RAO & $\begin{array}{l}\text { Five patients; two fresh } \\
\text { and three long- } \\
\text { standing cases; } \\
\text { cornea; both eyes }\end{array}$ \\
\hline
\end{tabular}
40 , and $80 \mathrm{~Hz}$ with 10-millisecond biphasic current pulses; 5 millisecond positive, thereafter 5 millisecond negative; periodically switching of the light, every 60-90 seconds

5-Milliseconds positive, thereafter 5-milliseconds negative biphasic pulses at $20 \mathrm{~Hz}, 30$ minutes once a week for 6 days, intensity $66 \%$ and $150 \%$ of EPT

VA; VF; electrophysiology; slit-lamp biomicroscopy; fundus examination; tonometry

Kinetic and static VFs; VA; full-field and mfERG

500-900- $\mu \mathrm{A}$, biphasic pulses at $20 \mathrm{~Hz}$, duration 30 minutes static perimetry with HFA
Results

TES improved VF; all functional parameters were improved or remained constant in $150 \%$ group; TES led to decreased desaturated color discrimination and VF mean sensitivity; no marked tendency was observed in $66 \%$ group

TES with a frequency of 20 $\mathrm{Hz}$ yielded the maximum PR; biphasic pulses with duration of $0.5-1.0$ millisecond and a frequency of 20-50 Hz were highly efficient for evoking phosphene

The VA increased in two subjects with NAION and in four subjects with TON

Improved function of retina in eyes with longstanding RAOs; the VA and mfERGs improved in two cases, and VF was improved in all three cases

Increased the chorioretinal blood flow in normal patients with mild effects on the systemic blood circulation and IOP

EEPR; BCVA; VF; phosphene All thresholds significantly higher in patients compared to healthy subjects; T1 and T2 were not correlated with VA, but related to the area and location of the remaining $\mathrm{VF} ; \mathrm{T} 1$ and $\mathrm{T} 2$ in RP eyes with EEPR was lower compared to RP eyes without an EEPR

EPTs differed between the disease groups; in all groups, EPTs were lowest at $20 \mathrm{~Hz}$; subjects with retinal diseases and across all frequencies exhibited higher EPTs than healthy patients, except in STG at $20 \mathrm{~Hz}$

The scotopic a-wave slopes increased in $150 \%$ treatment group; other parameters in all other groups were unchanged

BCVA; mfERGs; automated TES reforms the visual function in patients with branch RA0, primarily in long-standing cases

(table continues) 
Table 2 (continued)

\begin{tabular}{|c|c|c|c|c|c|c|}
\hline Reference & Disease & $\begin{array}{l}\text { Experimental population; } \\
\text { stimulation location }\end{array}$ & Control group & ES parameters & Evaluation method & Results \\
\hline $\begin{array}{l}\text { Ozeki et al, } \\
2013^{45}\end{array}$ & BVMD & $\begin{array}{l}\text { One patient; cornea; } \\
\text { right eye }\end{array}$ & - & $\begin{array}{l}\text { First treatment: } 250 \mu \mathrm{A} / \\
170 \mu \mathrm{A}, 20 \mathrm{~Hz}, 10 \\
\text { milliseconds/phase, } \\
\text { 30-minute duration, two } \\
\text { sessions with interval } \\
\text { of } 1 \text { month; second } \\
\text { treatment } 2 \text { years later: } \\
160 \mu \mathrm{A}, 20 \mathrm{~Hz}, 10 \\
\text { milliseconds, } 30 \text {-minute } \\
\text { duration, two sessions } \\
\text { with interval of } 1 \text { month }\end{array}$ & BCVA; VF; mfERG & $\begin{array}{l}\text { BCVA improved from } 20 / 40 \\
\text {-20/30 after } 1 \text { month, } \\
\text { and from 20/40-20/25 } \\
\text { after } 6 \text { months; slight } \\
\text { improvements for VFs } \\
\text { and mfERGs; BCVA } \\
\text { decreased to } 20 / 70 \text { after } \\
2 \text { years; second } \\
\text { treatment: BCVA } \\
\text { improved to } 20 / 30 \text { after } \\
1 \text { month; no } \\
\text { improvements in VFs and } \\
\text { mfERGs }\end{array}$ \\
\hline $\begin{array}{r}\text { Xie et al, } \\
2011^{46}\end{array}$ & Normal-sighted & $\begin{array}{l}\text { Six patients; cornea; } \\
\text { right eye }\end{array}$ & - & $\begin{array}{l}\text { DTL-Plus corneal electrode } \\
\text { and ERG-Jet contact lens } \\
\text { electrode; } 1.5 \times \text { EPT } \\
\text { current amplitude, } \\
\text { 30-minute duration, } 2 \\
\text { Hz, 2-millisecond pulse } \\
\text { width; after every } 5 \\
\text { minutes of continuous } \\
\text { TES, stimulation turned } \\
\text { off for } 30 \text { seconds }\end{array}$ & PET imaging; FDG activity & $\begin{array}{l}\text { The results with both } \\
\text { corneal electrodes } \\
\text { demonstrated activation } \\
\text { of areas in visual cortex } \\
\text { that were related to the } \\
\text { reported phosphene } \\
\text { percept; ERG-Jet was } \\
\text { able to generate brighter } \\
\text { phosphene percept } \\
\text { compared to DTL-Plus; } \\
\text { the use of ERG-Jet } \\
\text { led to activation of } \\
\text { retinotopically mapped } \\
\text { primary visual cortex }\end{array}$ \\
\hline $\begin{array}{l}\text { Xie et al, } \\
2012^{47}\end{array}$ & RD & $\begin{array}{l}\text { Five patients; cornea; } \\
\text { right eye }\end{array}$ & $\begin{array}{c}\text { Five healthy } \\
\text { patients }\end{array}$ & $\begin{array}{l}\text { First, } 30 \text { minutes dark } \\
\text { adaptation; } 1.5 \times \text { EPT } \\
\text { current amplitude, } \\
\text { 2-millisecond pulses, } 2 \\
\text { Hz with interpulse of } 996 \\
\text { milliseconds, } 30 \text {-minute } \\
\text { duration; after every } 5 \\
\text { minutes of continuous } \\
\text { TES, stimulation turned } \\
\text { off for } 30 \text { seconds }\end{array}$ & PET imaging; FDG activity & $\begin{array}{l}\text { EPT current was higher in } \\
\text { RD patients compared } \\
\text { with control; in both } \\
\text { groups, TES and light } \\
\text { stimulation resulted } \\
\text { in activation of } \\
\text { retinotopically mapped } \\
\text { primary visual cortex }\end{array}$ \\
\hline
\end{tabular}

BCVA, best-corrected visual acuity; BVMD, best vitelliform macular dystrophy; CRD, cone-rod dystrophy; DTL, Dawson-Trick-Litzkow; EEPR, electrically evoked pupillary response; EPT, electrical phosphene threshold; ERG, electroretinogram; ES, electrical stimulation; FDG, ${ }^{18}$ F-fluorodeoxyglucose; HFA, Humphrey field analyzer; IOP, intraocular pressure; LSFG, laser speckle flowgraphy; mfERG, multifocal electroretinogram; NAION, nonarteritic ischemic optic neuropathy; PET, positron emission tomography; POAG, primary open-angle glaucoma; $P R$, pupillary reflex; RAO, retinal artery occlusion, RD, retinal degeneration; RP, retinitis pigmentosa; SBR, square blur rate; STG, Stargardt disease; TES, transcorneal electrical stimulation; TON, traumatic optic neuropathy; VA, visual acuity; VF, visual field; -, not reported.

patients, and the results demonstrated a provisional increase in visual function in some patients ${ }^{54}$; best-corrected visual acuity was found to be improved in patients with both wet and dry AMD. ${ }^{56}$

\section{Possible Mechanisms Underlying the Effects of ES}

Despite several studies showing promising results in both animal experiments and clinical studies, there are presently few reports on the mechanisms of action for ES, making this field still poorly understood. Today, we know that after injuries like optic nerve transection, directly injured RGCs are rapidly lost, and so far there are no treatments that can prevent this process. However, the secondary loss of RGCs whose axons were not directly damaged is considerable, ${ }^{57}$ and the neuroprotective effect of ES is significant in preventing this secondary apoptosis.

The suggested mechanisms that are believed to underlie the effects of TES are summarized and presented in Figure 2. As in other tissues, it is believed that TES increases the expression of neurotrophic factors. It has been suggested that TES increases the number of surviving RGCs in vivo, probably because of increased levels of IGF-1 produced by Müller cells. ${ }^{24,58}$ Another study demonstrated that the expression of mRNA encoding brain-derived neurotrophic factor increased and that intracellular protein levels were found in cultured Müller cells after TES, ${ }^{59}$ supported by Ni et al. ${ }^{26}$ The last study also demonstrated that the levels of ciliary nerve trophic factor and expression of $B c l-2$ were both increased after TES. ${ }^{26}$ In cultured retinal Müller cells, Sato et $\mathrm{al}^{60}$ showed that TES also increased the expression of fibroblast growth factor 2, which was also 
Table 3 Transorbital and Transpalpebral ES: Clinical Studies

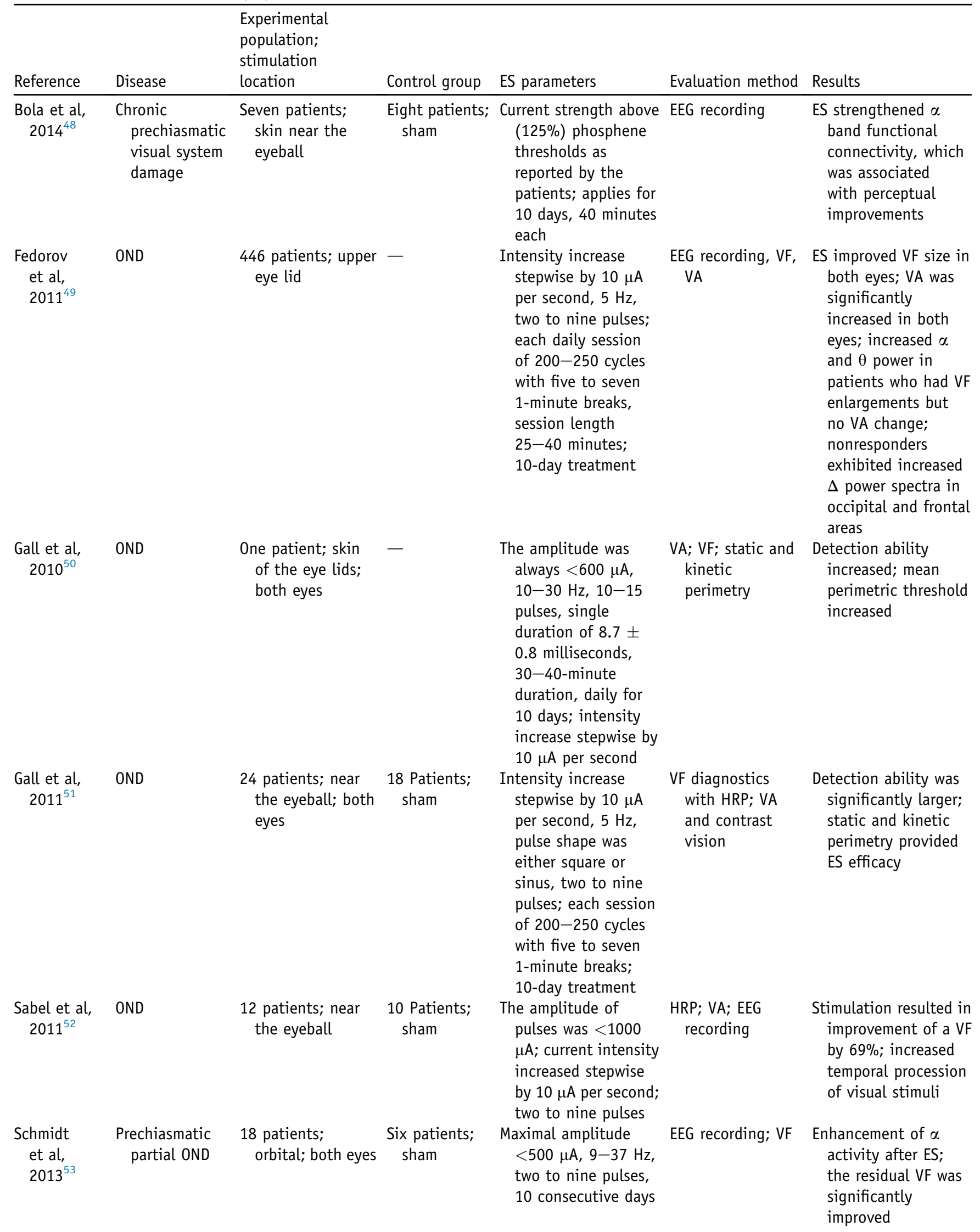

(table continues) 
Table 3 (continued)

\begin{tabular}{|c|c|c|c|c|c|c|}
\hline Reference & Disease & $\begin{array}{l}\text { Experimental } \\
\text { population; } \\
\text { stimulation } \\
\text { location }\end{array}$ & Control group & ES parameters & Evaluation method & Results \\
\hline $\begin{array}{l}\text { Anastassiou } \\
\text { et al, } \\
2013^{54}\end{array}$ & Dry AMD & $\begin{array}{l}12 \text { patients; } \\
\text { palpebral; both } \\
\text { eyes }\end{array}$ & $\begin{array}{l}10 \text { Patients; } \\
\text { sham }\end{array}$ & $\begin{array}{l}150-220 \mu \mathrm{A}, 5-80 \\
\mathrm{~Hz} ; 5 \text { consecutive } \\
\text { days, two sessions } \\
\text { on each day, every } \\
\text { session eight spots } \\
\text { (40 seconds/spot) }\end{array}$ & $\begin{array}{l}\text { VA testing before } \\
\text { treatment, after } \\
5 \text { days, after } 4 \\
\text { weeks, after } 6 \\
\text { months; macular } \\
\text { sensitivity } \\
\text { and fixation } \\
\text { stability with } \\
\text { microperimetry; } \\
\text { OCT }\end{array}$ & $\begin{array}{l}\text { Stimulation resulted in } \\
\text { improved VA in } 7 \text { of } \\
12 \text { patients, with } \\
\text { more than five } \\
\text { letters; contrast } \\
\text { sensitivity showed } \\
\text { a similar pattern }\end{array}$ \\
\hline $\begin{array}{l}\text { Chaikin et al, } \\
2015^{55}\end{array}$ & $\begin{array}{l}\text { Wet and dry } \\
\qquad \text { AMD }\end{array}$ & $\begin{array}{l}17 \text { patients; } 25 \\
\text { eyes with dry } \\
\text { type and } 6 \text { eyes } \\
\text { with wet type }\end{array}$ & - & $\begin{array}{l}150 \mu \mathrm{A} ; 3-162 \mathrm{~Hz} ; \\
35 \text {-minute session } \\
\text { once a week; average } \\
\text { number of } \\
\text { treatments was } 4.8\end{array}$ & $\begin{array}{l}\text { I0 pressure } \\
\text { measurements; } \\
\text { OCT; VA }\end{array}$ & $\begin{array}{l}\text { Significant } \\
\text { improvements in VA } \\
\text { in patients with dry } \\
\text { type, but not in wet } \\
\text { type; in dry-type } \\
\text { subjects, } 52 \% \text { of } \\
\text { patients exhibited } \\
\text { increased VA, and } \\
26 \% \text { deterioration }\end{array}$ \\
\hline $\begin{array}{l}\text { Shinoda } \\
\text { et al, } \\
2008^{56}\end{array}$ & $\begin{array}{l}\text { Wet and dry } \\
\text { AMD }\end{array}$ & $\begin{array}{l}21 \text { patients; } 16 \\
\text { (27 eyes) with } \\
\text { wet type and } 5 \\
\text { ( } 7 \text { eyes) with dry } \\
\text { type; palpebral; } \\
\text { both eyes }\end{array}$ & - & $\begin{array}{l}800 \mu \mathrm{A} \text {; one } 20 \text {-minute } \\
\text { session was } \\
\text { performed four times } \\
\text { per day, up to } 1 \\
\text { month; } 290 \mathrm{~Hz} \text { for } 1 \\
\text { minute, } 31 \mathrm{~Hz} \text { for } 2 \\
\text { minutes, } 8.9 \mathrm{~Hz} \text { for } \\
10 \text { minutes, } 0.28 \mathrm{~Hz} \\
\text { for } 7 \text { minutes }\end{array}$ & $\begin{array}{l}\text { BCVA before and } \\
\text { after } 4 \text { weeks; } \\
\text { slit-lamp } \\
\text { examination; } \\
\text { funduscopy; } \\
\text { fluorescein } \\
\text { angiography; } \\
\text { automated static } \\
\text { VF testing }\end{array}$ & $\begin{array}{l}\text { All of the patients } \\
\text { reported phosphene } \\
\text { perception; BCVA } \\
\text { improved, but did } \\
\text { not reach statistical } \\
\text { significance }\end{array}$ \\
\hline
\end{tabular}

$A M D$, age-related macular degeneration; BCVA, best-corrected visual acuity; EEG, electroencephalogram; ES, electrical stimulation; HRP, high-resolution perimetry; IO, intraocular; OND, optic nerve damage; OCT, optical coherence tomography; VA, visual acuity; VF, visual field; - , not reported.

confirmed by Ciavatta et $\mathrm{al}^{61}$ after implantation of microphotodiode arrays in Royal College of Surgeons rats. It has been suggested that L-type calcium channels probably control the release of these growth factors. This was demonstrated when an L-type voltage-dependent calcium channel blocker (nifedipine) was used to inhibit the upregulation of IGF-1 and brain-derived neurotrophic factor. $^{58,59}$

In primary microglia isolated from retinas of SpragueDawley rats, the inhibitory effects of ES on the secretion of IL-1 $\beta$ and tumor necrosis factor- $\alpha$ were confirmed, as well as favorable effects on the production of brain-derived neurotrophic factor and ciliary nerve trophic factor in Müller cells $^{35}$ and the down-regulation of the proapoptopic gene $\mathrm{Bax}^{26}$ Willmann et $\mathrm{al}^{34}$ also showed that TES led to clear changes in the expression of neuroprotective genes and that different genes may be expressed, depending on whether TES was applied to a healthy or diseased retina. Other effects of ES, investigated using a rat model of retinal ischemia, included increased secretion of glutamine synthetase from Müller cells, suggested to improve glutamate-mediated neuroexcitotoxicity. ${ }^{33}$ Furthermore, ES may also promote neuronal survival by increasing intracellular $\mathrm{Ca}^{2+}$ influx, which can cause neuronal cell depolarization and increase intracellular cyclic adenosine monophosphate levels. ${ }^{62}$ TES also was found to improve choroidal blood flow in normal-sighted patients, with mild effects on the systemic blood circulation and intraocular pressure. $^{40}$

The expression of retinal genes and the levels of retinal proteins associated with TES have recently been investigated. Willmann et $\mathrm{al}^{34}$ investigated the effect of TES on mRNA expression, retinal structure, and function. The authors demonstrated that TES induced differential the expression of 490 genes in the retina, including Bax and members of the tumor necrosis factor family. Physiological retinal function and intact structural properties were maintained for up to 35 hours after stimulation. ${ }^{34}$ A proteomic study by Kanamoto et $\mathrm{al}^{17}$ demonstrated that 25 proteins were up-regulated after TES, including cellular signaling 


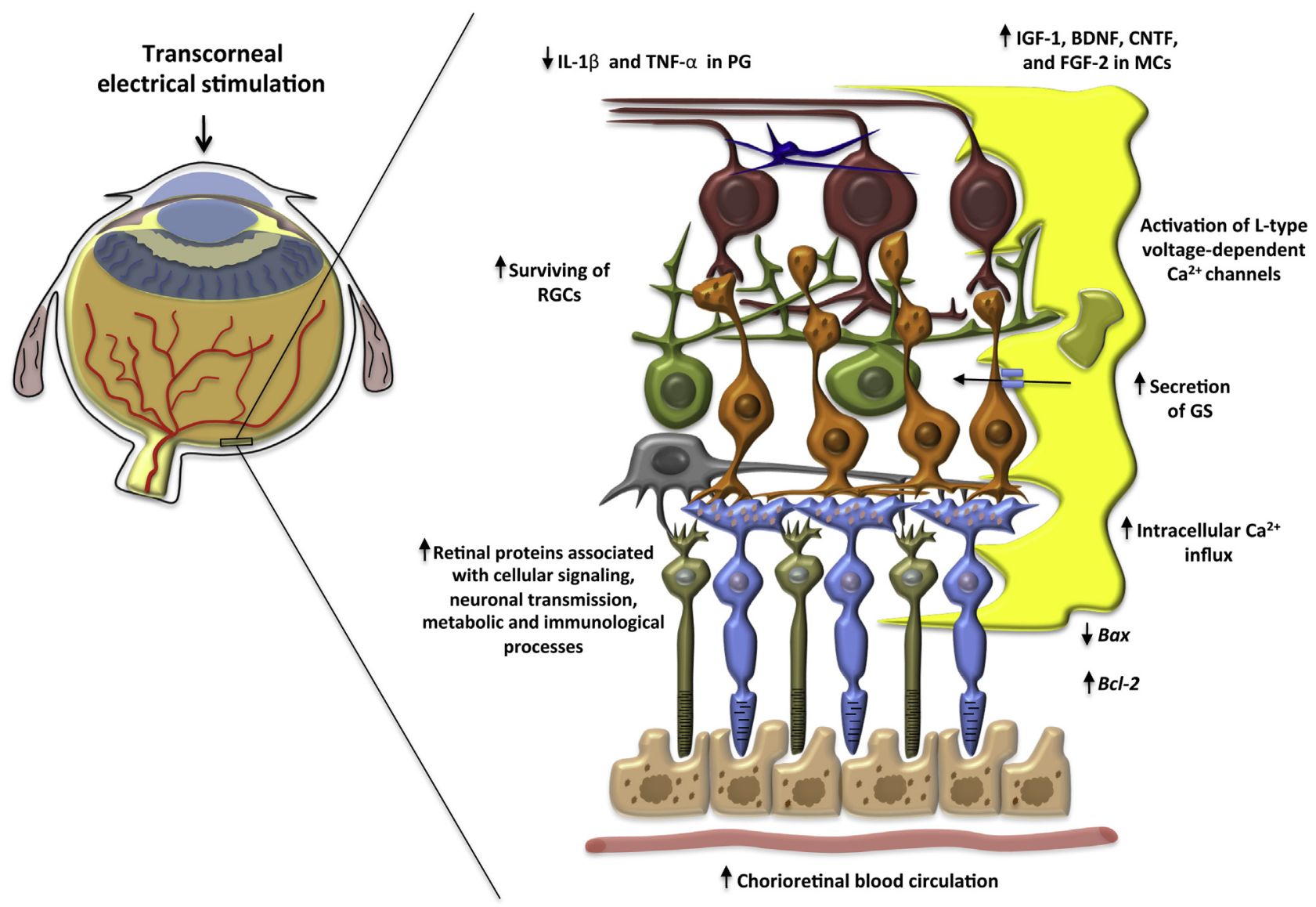

Figure 2 Possible neuroprotective mechanisms underlying the effects of transcorneal electrical stimulation. The cellular structure in the figure represents an enlarged image of the retina. BDNF, brain-derived neurotrophic factor; CNTF, ciliary nerve trophic factor; FGF, fibroblast growth factor; GS, glutamine synthetase; IGF-1, insulin-like growth factor 1 ; MCs, Müller cells; PG, primary microglia; RGCs, retinal ganglion cells; TNF- $\alpha$, tumor necrosis factor- $\alpha$.

proteins, proteins associated with neuronal transmission, metabolic proteins, immunological factors, and structural proteins. The pattern of differentially expressed proteins was altered at 30 minutes and 24 hours after TES, and the authors suggested that TES induced acute and chronic changes in protein expression. It was further proposed that the acute changes were direct and transient effects induced by electric shock to neural cells, whereas the chronic changes were indirect and secondary effects in mRNA expression or protein signaling pathways. ${ }^{17}$

\section{Complications and Safety Profile}

There are $>120$ articles on the use of invasive retinal prostheses for ES, making these approaches substantially more studied than other recently described, less invasive techniques. Both transpalpebral and transorbital approaches have the advantage of being minimally invasive (only touching the skin), with minor adverse effects reported. In a clinical study, it was reported that 1 of 21 patients developed contact dermatitis after transpalpebral ES. ${ }^{56}$ One investigation reported light frontal headache after transorbital ES in 3 of 18 patients. ${ }^{53}$ Furthermore, among 12 patients studied, 5 had some sensations while under the stimulation electrodes and 1 had spontaneous phosphene. ${ }^{52}$ TES touches only the cornea, which substantially reduces the risk of severe complications compared to retinal implants. The adverse effects were infrequent and mild for both DTL-Plus and ERG-Jet electrodes, with few patients reporting either foreignbody sensations with the DTL-Plus electrode ${ }^{36}$ or mild transient corneal punctate keratopathy on slit-lamp examination after the use of the ERG-Jet electrode. ${ }^{38}$ Schatz et $\mathrm{al}^{36}$ also reported minor conjunctiva irritation after intraocular pressure measurements. Other clinical TES studies reported slight corneal superficial punctuate keratopathy, ${ }^{41}$ transient superficial keratitis, ${ }^{39}$ and foreignbody sensations in three patients. ${ }^{43}$ Adverse effects after TES in animal studies have not been reported; however, one study addressed this concern and reported no negative effects up to 35 hours after application. ${ }^{34}$ All other methods will, by nature of their invasiveness, be prone to various complications, depending on the location. The excellent safety profile is likely to be substantially lower in future clinical settings. 


\section{Challenges and Future Perspectives}

A high number of recent reports demonstrate improvements in visual function by ES, which are expected to translate into large multicenter trials. The results from studies using different models of experimental animals have demonstrated favorable effects of ES on both the retina and optic nerve. However, the considerable problem with clinical studies so far has been the absence of randomization, a sham control, and inclusion of few patients. It is known that intraindividual changes in vision parameters vary significantly, common in neurorehabilitation; this makes it even more challenging in clinical trials. Another problem is that researchers all over the world experience the enormous advertising of stimulation prostheses and electrodes from many manufacturers because of considerable engineering and technical advances, making selection more difficult. As transpalpebral ES treatment is the only current treatment to show improved visual function in patients with AMD, more research to unravel the mechanisms behind its effects in an animal model is warranted. Such data are likely to yield clues on potential mechanisms of action using a transpalpebral approach as well as on how the advantageous effects on visual function in general can be further improved.

Stimulation parameters, such as current intensity, frequency, duration, and number of sessions, have been shown to vary in accordance with type of pathology and species. ${ }^{33,38}$ For example, in rats, the proposed current intensity of TES for protection of photoreceptors $(300 \mu \mathrm{A}, 3$ milliseconds per phase) is higher than that for survival of RGCs (100 $\mu \mathrm{A}, 1$ millisecond per phase). ${ }^{26,30}$ However, in humans, the threshold intensity required to elicit phosphene in both the central and peripheral visual fields is generally between 300 and $900 \mu \mathrm{A} .{ }^{44}$ Moreover, a study using positron emission tomography demonstrated that TES resulted in the activation of both retinotopographically matched primary visual cortex and visual perception in both normalsighted persons and subjects with retinal degenerative disease. ${ }^{44}$ However, the threshold current required to evoke phosphene is significantly lower in normal-sighted persons compared to patients with retinal degenerative disease. ${ }^{47}$ Optimizing the ES parameters needed to achieve the ideal balance between the beneficial effects and adverse effects is required.

\section{Conclusion}

ES with the purpose of restoring limited vision and/or treating blindness has undergone enormous scientific advances during the past 10 years. The amount of evidence for restoring visual function in various diseases using ES is rapidly increasing. We have currently reached a stage where ES has moved into clinical practice. Its favorable safety profile is likely to boost progress in ES for treating eye diseases. Still, there is a pressing need for a deeper understanding of the underlying mechanisms that govern clinical success. The optimization of stimulation parameters in animal studies, followed by large, prospective, clinical studies to explore the full potential of ES, is still required.

\section{Acknowledgment}

We thank Håkon Raanes for making Figure 1.

\section{References}

1. Leske MC, Heijl A, Hussein M, Bengtsson B, Hyman L, Komaroff E; Early Manifest Glaucoma Trial Group: Factors for glaucoma progression and the effect of treatment: the early manifest glaucoma trial. Arch Ophthalmol 2003, 121:48-56

2. Quigley HA, Broman AT: The number of people with glaucoma worldwide in 2010 and 2020. Br J Ophthalmol 2006, 90:262-267

3. Coleman HR, Chan CC, Ferris FL 3rd, Chew EY: Age-related macular degeneration. Lancet 2008, 372:1835-1845

4. Guadagni V, Novelli E, Piano I, Gargini C, Strettoi E: Pharmacological approaches to retinitis pigmentosa: a laboratory perspective. Prog Retin Eye Res 2015, 48:62-81

5. Maeda T, Dong Z, Jin H, Sawada O, Gao S, Utkhede D, Monk W, Palczewska G, Palczewski K: QLT091001, a 9-cis-retinal analog, is well-tolerated by retinas of mice with impaired visual cycles. Invest Ophthalmol Vis Sci 2013, 54:455-466

6. Solinis MA, Del Pozo-Rodriguez A, Apaolaza PS, RodriguezGascon A: Treatment of ocular disorders by gene therapy. Eur J Pharm Biopharm 2015, 95:331-342

7. Garg SJ, Federman J: Optogenetics, visual prosthesis and electrostimulation for retinal dystrophies. Curr Opin Ophthalmol 2013, 24 : 407-414

8. Dor H: Beiträge zur Electrotherapie der Augenkrankheiten. Albrecht von Graefes Arch Klin Exp Ophthalmol 1873, 19:532

9. Foerster O: Beitrage zur pathophysiologie der sehbahn und der spehsphare. J Psychol Neurol 1929, 39:435-463

10. Dobelle WH, Mladejovsky MG: Phosphenes produced by electrical stimulation of human occipital cortex, and their application to the development of a prosthesis for the blind. J Physiol 1974, 243: 553-576

11. Chow AY, Chow VY, Packo KH, Pollack JS, Peyman GA, Schuchard R: The artificial silicon retina microchip for the treatment of vision loss from retinitis pigmentosa. Arch Ophthalmol 2004, 122: 460-469

12. Lorach H, Marre O, Sahel JA, Benosman R, Picaud S: Neural stimulation for visual rehabilitation: advances and challenges. J Physiol Paris 2013, 107:421-431

13. Brindley GS, Lewin WS: The sensations produced by electrical stimulation of the visual cortex. J Physiol 1968, 196:479-493

14. Foik AT, Kublik E, Sergeeva EG, Tatlisumak T, Rossini PM, Sabel BA, Waleszczyk WJ: Retinal origin of electrically evoked potentials in response to transcorneal alternating current stimulation in the rat. Invest Ophthalmol Vis Sci 2015, 56:1711-1718

15. Henrich-Noack P, Lazik S, Sergeeva E, Wagner S, Voigt N, Prilloff S, Fedorov A, Sabel BA: Transcorneal alternating current stimulation after severe axon damage in rats results in "long-term silent survivor" neurons. Brain Res Bull 2013, 95:7-14

16. Henrich-Noack P, Voigt N, Prilloff S, Fedorov A, Sabel BA Transcorneal electrical stimulation alters morphology and survival of retinal ganglion cells after optic nerve damage. Neurosci Lett 2013, $543: 1-6$

17. Kanamoto T, Souchelnytskyi N, Kurimoto T, Ikeda Y, Sakaue H, Munemasa Y, Kiuchi Y: Proteomic study of retinal proteins 
associated with transcorneal electric stimulation in rats. J Ophthalmol 2015, 2015:492050

18. Ma Z, Cao P, Sun P, Li L, Lu Y, Yan Y, Chen Y, Chai X: Optical imaging of visual cortical responses evoked by transcorneal electrical stimulation with different parameters. Invest Ophthalmol Vis Sci 2014, 55:5320-5331

19. Mihashi T, Okawa Y, Miyoshi T, Kitaguchi Y, Hirohara Y, Fujikado T: Comparing retinal reflectance changes elicited by transcorneal electrical retinal stimulation with those of optic chiasma stimulation in cats. Jpn J Ophthalmol 2011, 55:49-56

20. Miyake K, Yoshida M, Inoue Y, Hata Y: Neuroprotective effect of transcorneal electrical stimulation on the acute phase of optic nerve injury. Invest Ophthalmol Vis Sci 2007, 48:2356-2361

21. Morimoto T, Fujikado T, Choi JS, Kanda H, Miyoshi T, Fukuda Y, Tano Y: Transcorneal electrical stimulation promotes the survival of photoreceptors and preserves retinal function in Royal College of Surgeons rats. Invest Ophthalmol Vis Sci 2007, 48:4725-4732

22. Morimoto T, Kanda H, Kondo M, Terasaki H, Nishida K, Fujikado T: Transcorneal electrical stimulation promotes survival of photoreceptors and improves retinal function in rhodopsin P347L transgenic rabbits. Invest Ophthalmol Vis Sci 2012, 53:4254-4261

23. Morimoto T, Kanda H, Miyoshi T, Hirohara Y, Mihashi T, Kitaguchi Y, Nishida K, Fujikado T: Characteristics of retinal reflectance changes induced by transcorneal electrical stimulation in cat eyes. PLoS One 2014, 9:e92186

24. Morimoto T, Miyoshi T, Matsuda S, Tano Y, Fujikado T, Fukuda Y: Transcorneal electrical stimulation rescues axotomized retinal ganglion cells by activating endogenous retinal IGF-1 system. Invest Ophthalmol Vis Sci 2005, 46:2147-2155

25. Morimoto T, Miyoshi T, Sawai H, Fujikado T: Optimal parameters of transcorneal electrical stimulation (TES) to be neuroprotective of axotomized RGCs in adult rats. Exp Eye Res 2010, 90:285-291

26. Ni YQ, Gan DK, Xu HD, Xu GZ, Da CD: Neuroprotective effect of transcorneal electrical stimulation on light-induced photoreceptor degeneration. Exp Neurol 2009, 219:439-452

27. Osako T, Chuman H, Maekubo T, Ishiai M, Kawano N, Nao IN: Effects of steroid administration and transcorneal electrical stimulation on the anatomic and electrophysiologic deterioration of nonarteritic ischemic optic neuropathy in a rodent model. Jpn J Ophthalmol 2013, 57:410-415

28. Rahmani S, Bogdanowicz L, Thomas J, Hetling JR: Chronic delivery of low-level exogenous current preserves retinal function in pigmented P23H rat. Vision Res 2013, 76:105-113

29. Schatz A, Arango-Gonzalez B, Fischer D, Enderle H, Bolz S, Rock T, Naycheva L, Grimm C, Messias A, Zrenner E, Bartz-Schmidt KU, Willmann G, Gekeler F: Transcorneal electrical stimulation shows neuroprotective effects in retinas of light-exposed rats. Invest Ophthalmol Vis Sci 2012, 53:5552-5561

30. Sergeeva EG, Fedorov AB, Henrich-Noack P, Sabel BA: Transcorneal alternating current stimulation induces EEG "aftereffects" only in rats with an intact visual system but not after severe optic nerve damage. J Neurophysiol 2012, 108:2494-2500

31. Sergeeva EG, Henrich-Noack P, Gorkin AG, Sabel BA: Preclinical model of transcorneal alternating current stimulation in freely moving rats. Restor Neurol Neurosci 2015, 33:761-769

32. Tagami Y, Kurimoto T, Miyoshi T, Morimoto T, Sawai H, Mimura O: Axonal regeneration induced by repetitive electrical stimulation of crushed optic nerve in adult rats. Jpn J Ophthalmol 2009, 53:257-266

33. Wang X, Mo X, Li D, Wang Y, Fang Y, Rong X, Miao H, Shou T: Neuroprotective effect of transcorneal electrical stimulation on ischemic damage in the rat retina. Exp Eye Res 2011, 93:753-760

34. Willmann G, Schaferhoff K, Fischer MD, Arango-Gonzalez B, Bolz S, Naycheva L, Rock T, Bonin M, Bartz-Schmidt KU, Zrenner E, Schatz A, Gekeler F: Gene expression profiling of the retina after transcorneal electrical stimulation in wild-type Brown Norway rats. Invest Ophthalmol Vis Sci 2011, 52:7529-7537

35. Zhou WT, Ni YQ, Jin ZB, Zhang M, Wu JH, Zhu Y, Xu GZ, Gan DK: Electrical stimulation ameliorates light-induced photoreceptor degeneration in vitro via suppressing the proinflammatory effect of microglia and enhancing the neurotrophic potential of Muller cells. Exp Neurol 2012, 238:192-208

36. Schatz A, Rock T, Naycheva L, Willmann G, Wilhelm B, Peters T, Bartz-Schmidt KU, Zrenner E, Messias A, Gekeler F: Transcorneal electrical stimulation for patients with retinitis pigmentosa: a prospective, randomized, sham-controlled exploratory study. Invest Ophthalmol Vis Sci 2011, 52:4485-4496

37. Fujikado T, Morimoto T, Kanda H, Kusaka S, Nakauchi K, Ozawa M, Matsushita K, Sakaguchi H, Ikuno Y, Kamei M, Tano Y: Evaluation of phosphenes elicited by extraocular stimulation in normals and by suprachoroidal-transretinal stimulation in patients with retinitis pigmentosa. Graefes Arch Clin Exp Ophthalmol 2007, 245: 1411-1419

38. Fujikado T, Morimoto T, Matsushita K, Shimojo H, Okawa Y, Tano Y: Effect of transcorneal electrical stimulation in patients with nonarteritic ischemic optic neuropathy or traumatic optic neuropathy. Jpn J Ophthalmol 2006, 50:266-273

39. Inomata K, Shinoda K, Ohde H, Tsunoda K, Hanazono G, Kimura I, Yuzawa M, Tsubota K, Miyake Y: Transcorneal electrical stimulation of retina to treat longstanding retinal artery occlusion. Graefes Arch Clin Exp Ophthalmol 2007, 245:1773-1780

40. Kurimoto T, Oono S, Oku H, Tagami Y, Kashimoto R, Takata M, Okamoto N, Ikeda T, Mimura O: Transcorneal electrical stimulation increases chorioretinal blood flow in normal human subjects. Clin Ophthalmol (Auckland, NZ) 2010, 4:1441-1446

41. Morimoto T, Fukui T, Matsushita K, Okawa Y, Shimojyo H, Kusaka S, Tano Y, Fujikado T: Evaluation of residual retinal function by pupillary constrictions and phosphenes using transcorneal electrical stimulation in patients with retinal degeneration. Graefes Arch Clin Exp Ophthalmol 2006, 244:1283-1292

42. Naycheva L, Schatz A, Rock T, Willmann G, Messias A, BartzSchmidt KU, Zrenner E, Gekeler F: Phosphene thresholds elicited by transcorneal electrical stimulation in healthy subjects and patients with retinal diseases. Invest Ophthalmol Vis Sci 2012, 53: $7440-7448$

43. Naycheva L, Schatz A, Willmann G, Bartz-Schmidt KU, Zrenner E, Rock T, Gekeler F: Transcorneal electrical stimulation in patients with retinal artery occlusion: a prospective, randomized, shamcontrolled pilot study. Ophthalmol Ther 2013, 2:25-39

44. Oono S, Kurimoto T, Kashimoto R, Tagami Y, Okamoto N, Mimura O: Transcorneal electrical stimulation improves visual function in eyes with branch retinal artery occlusion. Clin Ophthalmol (Auckland, NZ) 2011, 5:397-402

45. Ozeki N, Shinoda K, Ohde H, Ishida S, Tsubota K: Improvement of visual acuity after transcorneal electrical stimulation in case of Best vitelliform macular dystrophy. Graefes Arch Clin Exp Ophthalmol 2013, 251:1867-1870

46. Xie J, Wang GJ, Yow L, Cela CJ, Humayun MS, Weiland JD, Lazzi G, Jadvar H: Modeling and percept of transcorneal electrical stimulation in humans. IEEE Trans Biomed Eng 2011, 58: 1932-1939

47. Xie J, Wang GJ, Yow L, Humayun MS, Weiland JD, Cela CJ, Jadvar H, Lazzi G, Dhrami-Gavazi E, Tsang SH: Preservation of retinotopic map in retinal degeneration. Exp Eye Res 2012, 98: 88-96

48. Bola M, Gall C, Moewes C, Fedorov A, Hinrichs H, Sabel BA: Brain functional connectivity network breakdown and restoration in blindness. Neurology 2014, 83:542-551

49. Fedorov A, Jobke S, Bersnev V, Chibisova A, Chibisova Y, Gall C, Sabel BA: Restoration of vision after optic nerve lesions with noninvasive transorbital alternating current stimulation: a clinical observational study. Brain Stimul 2011, 4:189-201 
50. Gall C, Fedorov AB, Ernst L, Borrmann A, Sabel BA: Repetitive transorbital alternating current stimulation in optic neuropathy. NeuroRehabilitation 2010, 27:335-341

51. Gall C, Sgorzaly S, Schmidt S, Brandt S, Fedorov A, Sabel BA: Noninvasive transorbital alternating current stimulation improves subjective visual functioning and vision-related quality of life in optic neuropathy. Brain Stimul 2011, 4:175-188

52. Sabel BA, Fedorov AB, Naue N, Borrmann A, Herrmann C, Gall C: Non-invasive alternating current stimulation improves vision in optic neuropathy. Restor Neurol Neurosci 2011, 29:493-505

53. Schmidt S, Mante A, Ronnefarth M, Fleischmann R, Gall C, Brandt SA: Progressive enhancement of alpha activity and visual function in patients with optic neuropathy: a two-week repeated session alternating current stimulation study. Brain Stimul 2013, 6: 87-93

54. Anastassiou G, Schneegans AL, Selbach M, Kremmer S: Transpalpebral electrotherapy for dry age-related macular degeneration (AMD): an exploratory trial. Restor Neurol Neurosci 2013, 31: $571-578$

55. Chaikin L, Kashiwa K, Bennet M, Papastergiou G, Gregory W: Microcurrent stimulation in the treatment of dry and wet macular degeneration. Clin Ophthalmol (Auckland, NZ) 2015, 9:2345-2353

56. Shinoda K, Imamura Y, Matsuda S, Seki M, Uchida A, Grossman T, Tsubota K: Transcutaneous electrical retinal stimulation therapy for age-related macular degeneration. Open Ophthalmol J 2008, 2: 132-136

57. Levkovitch-Verbin H, Quigley HA, Martin KR, Zack DJ, Pease ME, Valenta DF: A model to study differences between primary and secondary degeneration of retinal ganglion cells in rats by partial optic nerve transection. Invest Ophthalmol Vis Sci 2003, 44: 3388-3393

58. Sato T, Fujikado T, Morimoto T, Matsushita K, Harada T, Tano Y: Effect of electrical stimulation on IGF-1 transcription by L-type calcium channels in cultured retinal Muller cells. Jpn J Ophthalmol 2008, 52:217-223

59. Sato T, Fujikado T, Lee TS, Tano Y: Direct effect of electrical stimulation on induction of brain-derived neurotrophic factor from cultured retinal Muller cells. Invest Ophthalmol Vis Sci 2008, 49: 4641-4646

60. Sato T, Lee TS, Takamatsu F, Fujikado T: Induction of fibroblast growth factor-2 by electrical stimulation in cultured retinal Mueller cells. Neuroreport 2008, 19:1617-1621

61. Ciavatta VT, Chrenek M, Wong P, Nickerson JM, Pardue MT: Growth factor expression following implantation of microphotodiode arrays in RCS rats. Invest Ophthalmol Vis Sci 2006, 47:3177

62. Morimoto T, Miyoshi T, Fujikado T, Tano Y, Fukuda Y: Electrical stimulation enhances the survival of axotomized retinal ganglion cells in vivo. Neuroreport 2002, 13:227-230 